Int. J. Curr. Res. Med. Sci. (2017). 3(3): 22-29

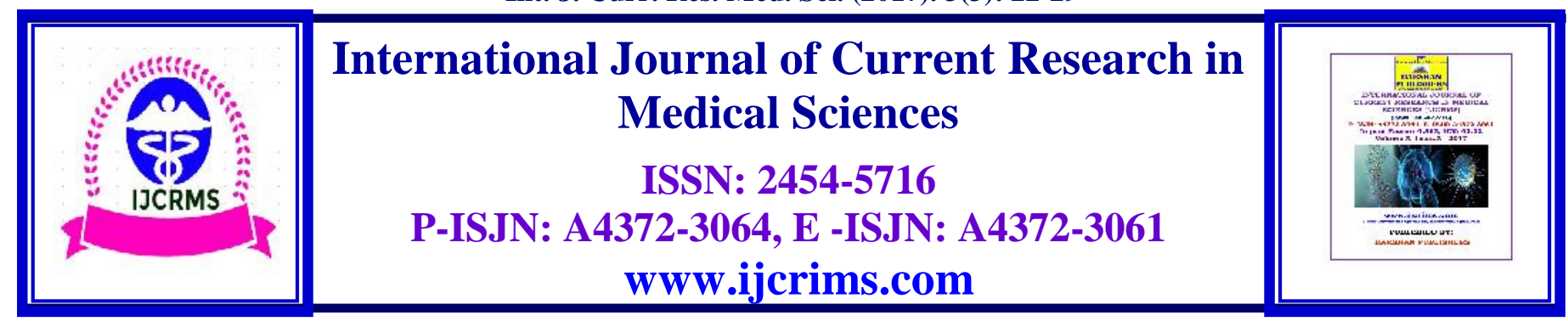

\title{
Prognostication of Perforation Peritonitis Cases Using Jabalpur Peritonitis Index
}

\author{
Sumitoj Singh", Sanjeev Sharma*, Satpal Hans", Nirbhaibir Singh"***, \\ Antariksh Gupta ${ }^{* * *}$, N.S Neki ${ }^{* * * * *}$, Gagandeep Singh Shergill \\ *Associate Professor, ${ }^{* *}$ Professor, ${ }^{* * *}$ Junior Resident, Department of General Surgery \\ Government Medical College, Amritsar, Punjab, India \\ ${ }^{* * * * *}$ Professor, ${ }^{* * * * *}$ Junior Resident, Department of Internal Medicine \\ Government Medical College, Amritsar, Punjab, India \\ Corresponding contributor: Dr Sumitoj Singh \\ Associate Professor, Department of General Surgery \\ Government Medical College, Amritsar, Punjab, India \\ E-mail-sumitoj@ rediffmail.com Phone no-91-9872041037
}

\section{Abstract}

Introduction: Perforation peritonitis is a common serious condition encountered in day to day surgical practice in any hospital. One of the reasons for high mortality in these conditions is lack of risk stratifications resulting in delay in providing adequate management, although a number of scoring systems are available to stratify these patients according to severity, but most of these cannot be used in developing countries like India due to their dependency on sophisticated investigation which are usually lacking in most parts of these countries. So a simple prognostic scoring system which could be easily reproduced without sophisticated investigations and also provide a reliable objective assessment of severity and operative risk is required.

Patients and methods: Fifty cases with diagnosis of peritonitis coming to Government Medical College, Amritsar were studied. Stratification of these patients was done according to Jabalpur peritonitis Index and their outcome was examined.

Results: Mortality steadily increases with increase in JPI score. Patients with JPI score of $>9$ and $<9$ had mortality rate of $2.5 \%$ and $60 \%$ respectively $(\mathrm{p}<0.01)$. Similarly patients with a score of $<9$ and $>9$ had morbidity of $30 \%$ and $80 \%$ respectively $(p<0.0001)$. Duration of pain $>24$ hours, age $>50$ years, mean SBP $<100 \mathrm{mmHg}$, serum creatinine $>1.5 \mathrm{mg} / \mathrm{dl}$, respiratory rate $>24 / \mathrm{min}$, heart rate $>110 / \mathrm{min}$ and feculent exudate intra operatively were found to be independently significant factors in predicting the mortality among the study population. For a score of 9 , the sensitivity was $85.7 \%$, specificity was $90.7 \%$ and positive predictive value for mortality is $40 \%$ and negative predictive value of $97.5 \%$.

Conclusion: This study proves that JPI scoring system is a simple and effective tool for assessing the morbidity and mortality in patients with peritonitis.

Keywords: Perforation; Jabalpur Peritonitis Index; Prognostication in perforation peritonitis. 


\section{Introduction}

Intestinal perforation peritonitis is a common condition encountered in surgical emergency department, loss of integrity of gastrointestinal tract with leakage of intestinal contents into the peritoneal cavity leads to an inflammatory response resulting in peritonitis. Complex interaction between various factors and early onset of specific therapeutic procedures determine the outcome of a case of perforation peritonitis. ${ }^{1}$ it also depends upon exact recognition of the seriousness of disease, an accurate assessment and classification of patient's risks ${ }^{2}$. In the last two decades several prognostic scores for abdominal sepsis have been evaluated, these allow stratification of patients according to severity, help in identification of patients at high risk and provide prognostic information. Various methods and scoring systems have been formulated over the year to identify the risks and prognosis in patients of perforation peritonitis. Some of the commonly used prognostic indices are.

\section{Mannheim peritonitis index8:}

It predicts outcome in patients with peritonitis. In this scoring system, age, duration of peritonitis, organ failure, diffuse peritonitis, site of perforation, level of exudates in peritoneal fluid are evaluated. Maximum score is 47 taking 27 as cut off point various studies found sensitivity to be around $60-80 \%$, specificity $90-100 \%$ and positive predictive value for mortality to be 90 $95 \%$.

\section{BOEY9:}

This scoring system predicts 30 days mortality in patients with peptic ulcer perforation. This system includes presentation $>$ or $<24$ hours comorbid conditions presence of pre-operative shock.

\section{HACETTEPE SCORE10:}

This score predicts 30 days mortality in patients with PPU( Peptic ulcer perforation)and it includes presence of serious medical illness, acute renal failure, white blood cell count, male gender as prognostic factors.

\section{PULP11:}

This score predicts 30 days mortality in patients with PPU and this score includes presentation more than 24 or less 24 hours, presence of preoperative shock; ASA( American society of anesthesiologists)score, active malignancy, presence of AIDS(Acquired immune deficiency syndrome), serum creatinine, liver failure $>130$ $\mathrm{mmol} / \mathrm{L}$.

\section{APACHE II12:}

This includes 2 parts: first one deal with acute physiology while second is concerned about chronic health evaluation. This system even though correctly measures severity of illness, but does not give any indication regarding the management modalities of the patient.

Most of these scoring systems have been in use since many years. None of these systems is a complete scoring system.

Major limitations of most of these scoring systems is their dependence on sophisticated investigation like $\mathrm{ABG}$, specialized computer softwares, computer operator etc. and difficulty in reproducibility. In a developing country like India where most of the critical care measures are unavailable and unaffordable by average citizens, it is vital that a simple and cost effective scoring system should be formulated which not only prognosticate accurately the outcome but should be easily reproducible.

To overcome these limitations a new prognostic scoring system was formulated which is based on simpler investigation which are easily available in surgical registers and can be easily reproduced. This is named Jabalpur peritonitis index. It was developed by Mishra et $\mathrm{al}^{3}$ based on the retrospective analysis of data from 140 patients with peritonitis, treated between Oct. 1999 and March 2011 in which 10 possible risk factors were considered, of these only 6 proved to be of prognostic relevance and were entered into the Jabalpur peritonitis index, classified according to their predictive values ${ }^{3}$. The information is collected at the time of admission and first laparotomy (Table 1 and 2). 
Int. J. Curr. Res. Med. Sci. (2017). 3(3): 22-29

Table 1- Jabalpur prognostic scoring system for perforation peritonitis

Summary of risk factors of Jabalpur peritonitis index and mortality

\begin{tabular}{|c|c|c|c|}
\hline Risk factors & Survivors & Expired & p-value \\
\hline \multicolumn{4}{|c|}{ Age (years) } \\
\hline$>50$ & 11 & 5 & \multirow{2}{*}{0.016} \\
\hline$<50$ & 32 & 2 & \\
\hline \multicolumn{4}{|l|}{$\begin{array}{c}\text { Pre-operative } \\
\text { intervals (hours) }\end{array}$} \\
\hline$<24$ & 7 & 0 & \\
\hline$>24$ & 36 & 7 & \\
\hline \multicolumn{4}{|c|}{ Mean systolic BP (mmHg) } \\
\hline$<100$ & 7 & 12 & \multirow{2}{*}{0.001} \\
\hline$>100$ & 0 & 31 & \\
\hline \multicolumn{4}{|c|}{ Heart rate (min) } \\
\hline$<110$ & 34 & 0 & \\
\hline$>110$ & 9 & 7 & \\
\hline \multicolumn{4}{|c|}{ Serum creatinine $(\mathrm{mg} / \mathrm{dl})$} \\
\hline$<1.4$ & 32 & 0 & \\
\hline$>1.4$ & 11 & 7 & \\
\hline
\end{tabular}

Table 2-Summary of risk factors of Jabalpur peritonitis index and mortality Jabalpur prognostic scoring system for peptic perforation:

\begin{tabular}{|c|c|c|c|c|c|c|c|}
\hline Factor & 0 & 1 & 2 & 3 & 4 & 5 & 6 \\
\hline $\begin{array}{c}\text { P-O Interval (hours) } \\
\text { Means systolic BP } \\
\text { (mmHg) }\end{array}$ & $70-109$ & - & $\begin{array}{c}50-69 \text { or } \\
110-129\end{array}$ & $130-159$ & $<49$ or $>160$ & - & - \\
\hline Heart rate (per minute) & $70-109$ & - & $\begin{array}{c}55-69 \text { or } \\
110-139\end{array}$ & $\begin{array}{c}40-54 \text { or } \\
140-179\end{array}$ & $<39$ or $>180$ & - & - \\
\hline $\begin{array}{c}\text { Serum creatinine } \\
(\mathrm{mg} / \mathrm{Dl})\end{array}$ & $0.6-1.4$ & - & $1.5-1.9$ & $2.0-3.4$ & $<39$ or $>180$ & - & - \\
\hline Age (years)* & $<45$ & & $45-54$ & $55-64$ & $>3.5$ & $65-74$ & $\begin{array}{c}75 \text { or } \\
\text { more }\end{array}$ \\
\hline
\end{tabular}

p-o interval $=$ perforation-operation interval

$*$ co-morbid illness, if present, is given score $=5$

\section{Patients and Methods}

This was a prospective study done in 50 patients with peritonitis who presented at Government Medical College, Amritsar between October 2014 to October 2016. Study was conducted after taking required clearance from ethics committee of the institute.

The study was done after obtaining a detailed history, complete general physical examination and systemic examination. The patients were subjected to relevant investigations like X-Ray erect abdomen, Chest X-Ray, Ultrasound Abdomen, and routine investigations like $\mathrm{Hb}, \mathrm{BT}$, CT, TLC, DLC, RBS, urea, creatinine.

All investigations and surgical procedures were carried out with proper informed written consent as appropriately. The data regarding patient particulars, diagnosis, investigations, and surgical procedures was collected in a specially designed case recording form. Statistical analysis was done using SPSS 22, IBM. 


\section{Inclusion criteria:}

Patients with clinical suspicion and investigatory support for the diagnosis of Peritonitis irrespective of their age and sex.

\section{Exclusion criteria:}

Patients with

1. Associated vascular, neurogenic injuries.

\section{Aims and objectives:}

This study attempted to evaluate the prognostic value of Jabalpur Peritonitis Index scoring system in patients with peritonitis, to assess it as a clinical tool in stratifying these patients according to individual surgical risk.

\section{Results}

This study was done in 50 patients with peritonitis who presented at Government Medical College, Amritsar between October 2014 to October 2016.In this study, majority of patients 25/50 belonged to age group of 21 to 50 years, youngest was 8 years old female, while the oldest was 80 years old male mean age was 40.3 years. There were $44 / 50(88 \%)$ male and $6 / 50(12 \%)$ female patients in this study with male to female ratio being 7.3:1. It was found that mortality rate is $2 / 34(5.88 \%)$ for patients $<50$ years and $5 / 16(31.25 \%)$ for patients $>50$ years.

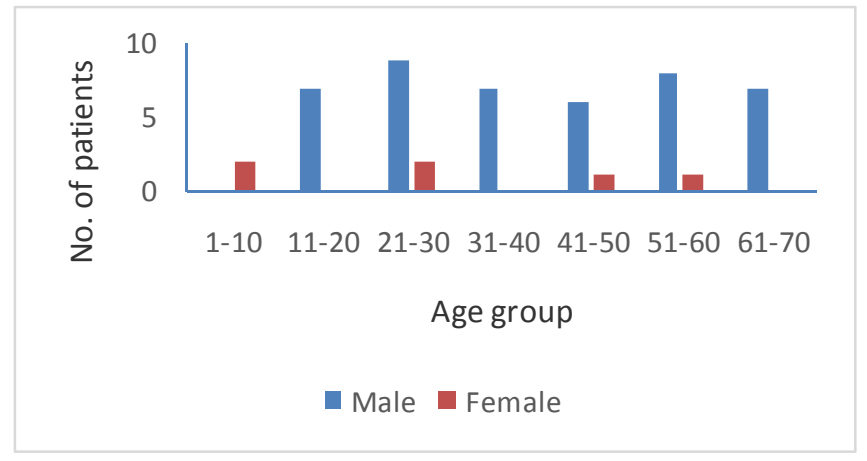

Figure 1-Age and sex distribution

In this study 3 patients had traumatic perforation and rest 47 had non traumatic perforation. Among traumatic 1 was dedunal and 2 were jejunal perforations. In non traumatic group Ileal perforation $20 / 47(42.5 \%)$ was the most frequent aetiology of peritonitis, followed by gastric perforation 18/47(38.5\%) Appendicular perforation $3 / 47(6.5 \%)$ and caecal perforation $3 / 47(6.5 \%)$. jejunal perforation $2 / 47(4 \%)$ and duodenal perforation $1 / 50(2 \%)$ were other causes. According to cause peptic ulcer perforation $19 / 47(41 \%)$ was most commom followed by typhoid perforation 17/47(37\%), appendicular gangrene $3 / 47(6 \%)$, tubercular perforation $3 / 47(6 \%)$ malignant perforation $2 / 47(4 \%)$, ischaemic intestinal perforation $2 / 47(4 \%)$ and meckel's diverticulitis perforation $1 / 47(2 \%)$ were other causes.
Majority of the patients 43/50 presented in hospital after 24 hours (86\%) and most commonly during 25-72 hours 30/50(60\%). Mortality of patients was highest $2 / 2(100 \%)$ among those presented in the hospital after 120 hours, and it was $2 / 3(66.6 \%)$ for those who presented between 97-120 hours, there was no mortality in patients who presented within 24 hours. In this study mortality of patients having serum creatinine levels less than $1.4 \mathrm{mg} / \mathrm{dl}$ was nil and those with more than $1.4 \mathrm{mg} / \mathrm{dl}$ was $7 / 18(38.8 \%)$. The mortality of patients who had mean systolic blood pressure $>100 \mathrm{mmHg}$ was nil and those with mean systolic blood pressure $<100 \mathrm{mmHg}$ was $7 / 18(38.8 \%)$. Mortality rate of patients with heart rate of $>110 / \mathrm{min}$ was $7 / 16(43.75 \%)$ and in those with heart rate $<110 /$ min was nil. 
This study showed that mortality steadily increases with increase in JPI score showing statistically significant results $(\mathrm{p}<0.001$, fig. 2$)$.

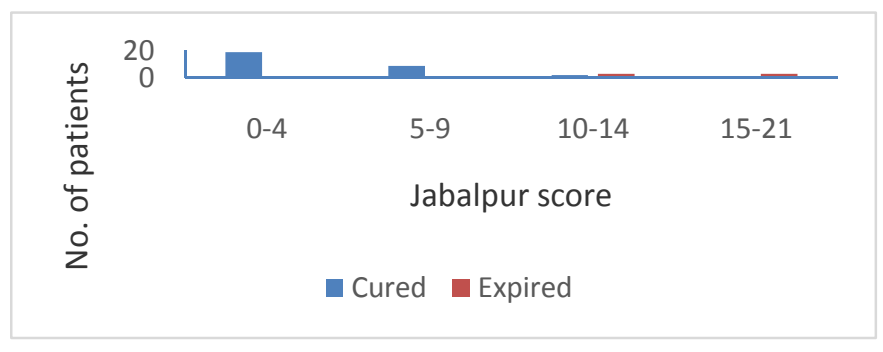

Figure 2-mortality and jabalpur peritonitis index score

Various postoperative complications seen in the study group were wound sepsis, wound dehiscence, acute renal failure, pneumonitis, acute respiratory distress syndrome and shock. The study also showed that morbidity steadily increases with increasing JPI score $(\mathrm{p}<0.001$, Fig.3).

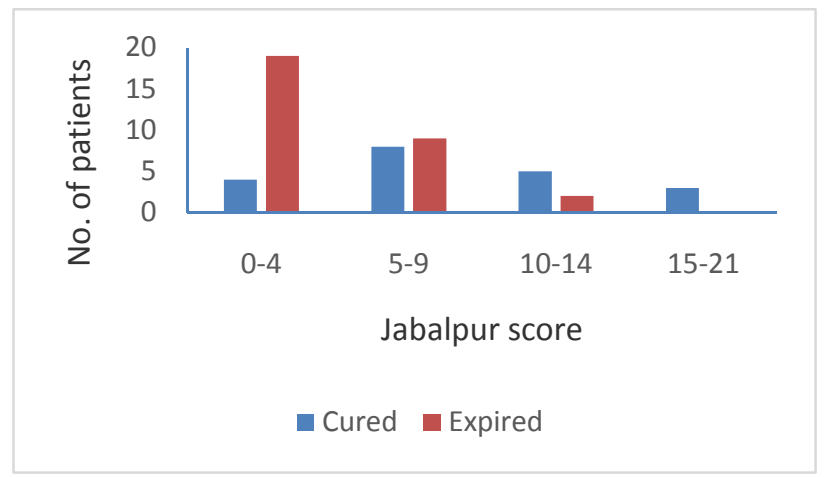

Figure 3-mortality and jabalpur peritonitis index score

For a score of 9, the sensitivity was $85.7 \%$, specificity was $90.7 \%$ and positive predictive value for mortality is $40 \%$ and negative predictive value of $97.5 \%$.

Table 3-Sensitivity and specificity of Jabalpur peritonitis index score 9.

\begin{tabular}{|c|c|c|c|}
\hline $\begin{array}{c}\text { Jabalpur peritonitis } \\
\text { index score }\end{array}$ & Expired & Cured & Total \\
\hline$>9$ & 6 & 4 & 10 \\
\hline$<9$ & 1 & 39 & 40 \\
\hline
\end{tabular}

\section{Discussion}

Perforation peritonitis is one of the common emergency condition encountered in surgical department worldwide. If not managed properly this is usually a morbid condition. Loss of integrity of gastrointestinal tract with consecutive leak of the intestinal contents into the peritoneal cavity leading to an inflammatory response resulting in peritonitis. This resulting phenomena is localized equivalent of the systemic inflammatory response which is usually seen after any trigger of inflammation ${ }^{4},$. . Various factors like age, sex interval between perforation and operation site of perforation, extent of peritonitis and delay in surgical intervention are associated 
with increased morbidity and mortality ${ }^{2}$. A successful outcome depends upon exact recognition of the seriousness of the disease, an accurate assessment and classification of patients according to severity ${ }^{2}$.

Despite the surgical treatment and sophisticated ICUs, latest generation antibiotics and a better understanding of pathophysiology of peritonitis mortality rate are still very high, i.e., 10-20\% even in good institutions. Dickson and Cole recorded $56 \%$ mortality rate in 38 case study. 4 Butler found the overall mortality rate to be $70 \%$ with medical treatment and $26 \%$ with surgery. ${ }^{6,7}$ Early prognostic evaluation is necessary to select high risk patients for more aggressive therapeutic procedures such as radical debridement, lavage systems, open management, and planned reoperations.

Classification of the severity of disease includes the definition of groups by risk, and it is of confirmed value in both controlled and uncontrolled clinical trials. An accurate risk index classification is one of the method to settle a standard of comparison between groups of patient and different treatment methods which would allow further prospective adequate comparative study.

Various methods and scoring systems have been formulated over the year to identify the risks and prognosis in patients of perforation peritonitis. Some of the commonly used prognostic indices are: Mannheim peritonitis index, BOEY, HACETTEPE SCORE, PULP, APACHE II etc.

Most of these scoring systems have been in use since many years. None of these systems is a complete scoring system.

Major limitations of most of these scoring systems is their dependence on sophisticated investigation and difficulty in reproducibility. In a developing country like India where most of the critical care measures are unavailable and unaffordable by average citizens, it is vital that a simple and cost effective scoring system should be formulated which not only prognosticate accurately the outcome but should be easily reproducible.

To overcome these limitations a new prognostic scoring system was formulated which is based on simpler investigation which are easily available in surgical registers and can be easily reproduced. This is named Jabalpur peritonitis index.

Jabalpur peritonitis index predicts mortality in patients of perforation peritonitis and is based on time from perforation to operation, mean systolic blood pressure preoperatively, heart rate, serum creatinine, age and associated co-morbid illness.

In 2003, Mishra et al studied 140 consecutive patients (mean age 38.9; range $15-80$ years), with perforated pre-pyloric / duodenal ulcer and found that mean duration of hospital stay was 13.4 days (range 1 to 54), fifteen patients died, fifty one patients had 89 complications. On multiple regression analysis, age, perforation, operation interval, pre-operative shock, co-morbid illness, heart rate and serum creatinine were associated with death. Mortality rate was 3\% (1 of 38) when perforation to operation interval was $0-24$ hours, $4.5 \%$ (3 of 67 ) when interval was 25-72 hours. $17 \%$ (3 of 23) when the interval was 97-120 hours, and $80 \%$ (4 of 5 ) when the perforation operation interval was $>120$ hours. The Jabalpur score (range 0-21) correlated with mortality and morbidity. No patient with Jabalpur score of 0-4 died, whereas all patients with score exceeding 15 died.

Survivors had a lower mean score $4.9 \pm 3.3$ than non survivors $12.5 \pm 4.2 ; \mathrm{p}<0.001$, using a score of 9 as cut-off for predicting mortality. The Jabalpur system had a sensitivity of $87 \%$ (13/15), specificity of $85 \%(106 / 125)$. Positive predicting value of $41 \%(13 / 32)$ and negative predictive value of $98 \%$ (106/108). Jabalpur score was compared with Hacettepe, Boey's modified MPI and modified APACHE-II scores in all the patients. Comparison of ROC curves showed the performance of all scores to be comparable ${ }^{3}$

In 2010 Hemant et al studied 82 consecutive typhoid ileal perforation patients, mean age was 30.02 years (range 14-62). There were six times 
as many males as females (70:12). The mean duration of hospital stay was 15.18 days (range 148). Seventeen $(20.7 \%)$ patients died and 43 (52.4\%) patients had complications for all there patients JPI was calculated. The mortality rate was $0 \%$ when perforation operation interval was $<24$ hours, $11 \%(\mathrm{~m}=4 / 36)$ when interval was 25 72 hours, $55.5 \%(\mathrm{n}=5 / 9)$ between $97-120$ hours and $100 \%(n=4 / 4)$ when interval was $>120$ hours. Overall the JPS correlated with morbidity and mortality in TIP(typhoid ileal perforation) patients and as the score increased, so did the morbidity and mortality. ${ }^{13}$

Jagdeesh T.S et al in 2013 studied 94 patients of peritonitis, peptic $(n=55)$ followed by ileal, colonic and appendicular perforations were the commonest cause of peritonitis, 13/94 died; ileal perforations had the highest $(n=6 / 34,17.6 \%)$ mortality. When correlated with mortality univariate analysis showed JPI to be accurate prognostic marker $(\mathrm{p}<0.0000){ }^{14}$

The study proves that JPI scoring system is a simple and effective tool for assessing morbidity and mortality in patients with peritonitis with statistically significant results. Age $>50$ years, duration of pain $>24$ hours, mean SBP $<100$ $\mathrm{mmHg}$, serum creatinine $>1.4 \mathrm{mg} / \mathrm{dl}$, respiratory rate $>24 /$ mins, heart rate $>110 / \mathrm{min}$ and falculant exudate intraoperatively were found to be independently significant factors in predicting the mortality among the study group.

\section{Conclusion}

Increasing JPI score predicts poor prognosis, patients with higher score usually have a poor outcome and higher complication rate, such patients should be monitored closely in a specialized unit with intensive monitoring of vitals. To conclude, JPI appears to be simple and reliable tool in predicting the outcome in cases of peritonitis but still more work needs to be done before this could be widely adopted as a standard tool in prognosticating perforation peritonitis cases.

\section{References}

1. Seiler CA, Brügger L, Forssmann U, Baer HU, Büchler MW. Conservative surgical treatment of diffuse peritonitis. Surgery. 2000;127(2):178-84.

2. o Mannheim PD. Prediction of death using the mannheim peritonitis index in oncologic patients. Revista Brasileira de Cancerologia. 2001;47(1):63-8.

3. Mishra A, Sharma D, Raina VK. A simplified prognostic scoring system for peptic ulcer perforation in developing countries. Indian $\mathbf{J}$ Gastroenterol. 2003; 22(2):49-53.

4. Goris RJ, te Boekhorst TP, Nuytinck JK, Gimbrère JS. Multiple-organ failure: generalized autodestructive inflammation?. Archives of Surgery. 1985;120(10):1109-15..

5. Marshall J, Sweeney D. Microbial infection and the septic response in critical surgical illness: Sepsis, not infection, determines outcome. Archives of Surgery. 1990;125(1):17-23.

6. Dickson JA, Cole GJ. Perforation of the terminal ileum. A review of 38 cases. British Journal of Surgery. 1964;51(12):893-7.

7. Butler T, Knight J, Nath SK, Speelman P, Roy SK, Azad MA. Typhoid fever complicated by intestinal perforation: a persisting fatal disease requiring surgical management. Review of Infectious Diseases. 1985;7(2):244-56.

8. Wacha H, Linder MM, Feldmann U, Wesch G, Gundlach E, Steifensand RA. Mannheim peritonitis index-prediction of risk of death from peritonitis: construction of a statistical and validation of an empirically based index. Theor Surg. 1987;1(169):77.

9. Boey J, Choi SK, Poon A, Alagaratnam TT. Risk stratification in perforated duodenal ulcers. A prospective validation of predictive factors. Annals of surgery. 1987;205(1):22.

10.Altaca G, Sayek I, Onat D, Cakmakci M, Kamiloğlu S. Risk factors in perforated peptic ulcer disease: comparison of a new score system with the Mannheim Peritonitis Index. The European journal of surgery= Acta chirurgica. 1992;158(4):217-21. 
11.Møller $\mathrm{MH}$, Engebjerg MC, Adamsen S, Bendix J, Thomsen RW. The Peptic Ulcer Perforation (PULP) score: a predictor of mortality following peptic ulcer perforation. A cohort study. Acta Anaesthesiologica Scandinavica. $2012 ; 56(5): 655-62 .$.

12.Knaus WA, Draper EA, Wagner DP, Zimmerman JE. APACHE II: a severity of disease classification system. Critical care medicine. 1985;13(10):818-29.

13.Singh H, Mishra A, Sharma D, Somashekar U. A simple prognostic scoring system for typhoid ileal perforation peritonitis. Tropical doctor. 2010;40(4):203-7

14.Jagdeesh TS, Mishra A, Saxena A, Sharma D. Eosinopenia as a prognostic marker in patients with peritonitis. ISRN Infectious Diseases 2012; 28:2013.

\begin{tabular}{|c|l|}
\hline \multicolumn{2}{|c|}{ Access this Article in Online } \\
\hline Quick Response Code & Website: \\
\hline & Www.ijcrims.com \\
\hline & Subject: \\
\hline
\end{tabular}

How to cite this article:

Sumitoj Singh, Sanjeev Sharma, Satpal Hans, Nirbhaibir Singh, Antariksh Gupta, N.S Neki, Gagandeep Singh Shergill. (2017). Prognostication of Perforation Peritonitis Cases Using Jabalpur Peritonitis Index. Int. J. Curr. Res. Med. Sci. 3(3): 22-29.

DOI: http://dx.doi.org/10.22192/ijcrms.2017.03.03.004 